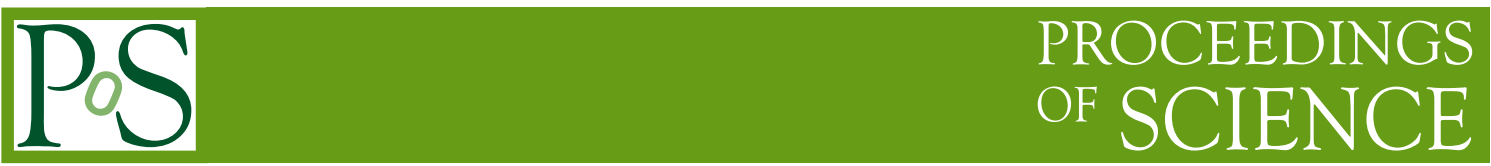

\title{
SM Higgs searches by CMS at the LHC
}

\author{
M. Kirsanov*i \\ INR Moscow \\ E-mail: Mikhail.Kirsanovecern.ch
}

Results are presented from searches for the standard model (SM) Higgs boson in proton-proton collisions at $\sqrt{s}=7$ and $8 \mathrm{TeV}$ in five decay modes: $\gamma \gamma, b b, \tau \tau, W W$, and $\mathrm{ZZ}$. The analysed data correspond to integrated luminosities of up to $5.1 \mathrm{fb}^{-1}$ at $7 \mathrm{TeV}$ and up to $12.2 \mathrm{fb}^{-1}$ at $8 \mathrm{TeV}$. The search is performed in the mass range $110-1000 \mathrm{GeV}$. No evidence is found for a significant excess of events that could be attributed to an additional particle, beyond the recently observed boson with a mass near $125 \mathrm{GeV}$. The significance of the latter is now $6.9 \sigma$. Its mass is measured to be $125.8 \pm 0.4$ (stat) \pm 0.4 (syst) The event yields obtained by the different analyses targeting specific decay modes and production mechanisms are consistent with those expected for the SM Higgs boson. The best-fit signal strength for all channels combined, expressed in units of the SM Higgs boson cross section, is $0.88 \pm 0.21$. The consistency of the couplings of the observed boson with those predicted for the SM Higgs boson is tested in various ways, and no significant deviations are found. Under the assumption that the observed boson has spin zero, the data disfavour the psudo-scalar hypothesis with a $C L_{s}$ value of $2.4 \%$.

LHC on the March - IHEP-LHC,

20-22 November 2012

Institute for High Energy Physics, Protvino,Moscow region, Russia

\footnotetext{
* Speaker.

${ }^{\dagger}$ On behalf of the CMS Collaboration
} 


\section{Introduction}

The Standard Model (SM) [1], [2] of particle physics has been very successful in explaining experimental data. In the SM the Higgs mechanism is postulated in order to explain the origin of the masses of the $\mathrm{W}$ and $\mathrm{Z}$ bosons that arise from electroweak symmetry breaking, which leads to an additional scalar field whose quantum, the Higgs boson, should be experimentally observable [3], [4].

The mass of the Higgs boson is not fixed in the Standard Model. The constraints on it are derived from the following considerations [5]:

- "Unitarity": Higgs boson exchange allows to avoid unphysical W - W scattering probability at high energies if $M_{H}<800 \mathrm{GeV}$

- "Triviality": Higgs boson self-coupling becomes too strong (so that perturbative methods are not applicable) if its mass is higher than some value depending on the cut-off scale $\Lambda$. This value has significant uncertainties, approximately it is given by $M_{H}^{2}<4 \pi^{2} v^{2} /(3 \ln (\Lambda / v))$.

- "Stability" of the vacuum: potential $V(\phi)$ has only one absolute minimum. Requires $M_{H}^{2}>$ $m_{t}^{4} \ln (\Lambda / v) / \pi^{2} v^{2}$

Here $v$ is the vacuum-expectation value of the Higgs field and $\Lambda$ is the cut-off scale.

In various extentions of the SM the Higgs sector can be more complicated, with additional observable particles.

The LHC and its major experiments were conceived and built to explore in depth the multi-TeV region and solve in a way or in another this major puzzle of particle physics. Excellent performance of the LHC in 2011 and 2012 allowed to the experiments to collect enough data to reach sensitivity exceeding or close to the SM Higgs boson cross sections. The teams of physicists of the ATLAS and CMS experiments managed to quickly perform the analysis of data in complicated conditions of pile-up (multiple proton-proton interactions in one bunch crossing, 10 - 15 on the average) and high backgrounds (cross sections up to $10^{11}$ of the Higgs boson cross sections). As a result of this work a boson with a mass of about $125 \mathrm{GeV}$ with properties compatible with the SM predictions for the Higgs boson was recently observed [6] [7].

The results of the continuation of the searches for particles similar to the SM Higgs boson in the CMS experiment and further studies of the previously observed boson are presented in this paper.

\section{The CMS detector}

A detailed description of the CMS detector can be found elsewhere [8]. The main features and those most pertinent to this analysis are described below. The central feature is a superconducting solenoid, $13 \mathrm{~m}$ in length and $6 \mathrm{~m}$ in diameter, which provides an axial magnetic field of $3.8 \mathrm{~T}$. The bore of the solenoid is instrumented with particle detection systems. The steel return yoke outside the solenoid is instrumented with gas detectors used to identify muons. Charged particle trajectories are measured by the silicon pixel and strip tracker, with full azimuthal coverage within 
$|\eta|<2.5$, where the pseudorapidity $\eta$ is defined as $\eta=-\ln [\tan (\Theta / 2)]$, with $\Theta$ being the polar angle of the trajectory of the particle with respect to the counterclockwise beam direction. A leadtungstate crystal electromagnetic calorimeter (ECAL) and a brass/scintillator hadron calorimeter (HCAL) surround the tracking volume and cover the region $|\eta|<3$. The ECAL barrel extends to $|\eta|=1.48$. A lead/silicon-strip preshower detector is located in front of the ECAL endcap. A steel/quartz-fibre Cherenkov forward calorimeter extends the calorimetric coverage to $|\eta|<5$.0. In the region $|\eta|<1.74$, the HCAL cells have widths of 0.087 in both pseudorapidity and azimuth $(\phi)$. In the $(\eta, \phi)$ plane, and for $|\eta|<1.48$, the HCAL cells map on to 5x5 ECAL crystal arrays to form calorimeter towers projecting radially outwards from points slightly offset from the nominal interaction point. In the endcap, the ECAL arrays matching the HCAL cells contain fewer crystals. Calibration of the ECAL uses $\pi^{0} \mathrm{~s}, W \rightarrow e v$, and $Z \rightarrow e e$. Deterioration of transparency of the ECAL crystals due to irradiation during the LHC running periods and their subsequent recovery is monitored continuously and corrected for using light injected from a laser and LED system.

\section{SM Higgs boson production and decays}

There are four main mechanisms for Higgs boson production in pp collisions. The gluongluon fusion mechanism has the largest cross section, followed in turn by vector boson fusion (VBF), associated $\mathrm{WH}$ and $\mathrm{ZH}$ production, and production in association with top quarks, $t \bar{t} H$. The Higgs boson decay modes are determined by its nature, in particular by the fact that its coupling to elementary particles (except gauge bosons, for which the dependency is more complicated) is proportional to their masses. This means that it prefers to decay to heavy particles. For the same reason the brancing ratios depend strongly on the Higgs boson mass $m_{H}$, as shown in Figure 1. These peculiarities of the Higgs boson decays and comparably small production cross sections render its searches rather complicated, in particular for some ranges of $m_{H}$, for example below 120 $\mathrm{GeV}$. The strategy of the searches for the Higgs boson are in turn determined by the peculiarities mentioned above. The decays modes most relevant to the searches at the LHC are listed in Table 1.

There are three types of independent theoretical uncertainties on Higgs boson production: uncertainties associated with (i) parton density functions (PDFs), (ii) incomplete perturbative calculations (also known as QCD scale uncertainties), and (iii) the treatment of the finite width of the Higgs boson. All these uncertainties are taken from Ref. [9].

\section{Higgs boson searches in the CMS detector}

The CMS analyses and references to the corresponding public documentation are shown in Table 2.

The $W W^{(*)} \rightarrow 2 l 2 v$ analysis [10] searches for an excess of events with two leptons of opposite charge, large $E_{T}^{\text {miss }}$, and up to two jets. Events are divided into six categories, with different background compositions and signal-to-background ratios. For events with no jets, the main background stems from non-resonant WW production; for events with one jet, the dominant backgrounds are from WW and top-quark production. The events are split into same-flavour and different-flavour dilepton sub-channels, since the background from Drell- Yan production is much larger for the same-flavour dilepton events. The two-jet category is optimized to take advantage of the VBF 
Higgs boson production signature. The main back ground in this channel is from top-quark production. In the $7 \mathrm{TeV}$ analysis, the same-flavour and different-flavour categories with 2 jets are merged into one. To improve the separation of signal from backgrounds in the $7 \mathrm{TeV}$ analysis, MVA classifiers are trained for a number of Higgs boson masses. The search is made for an excess of events in the output distributions of the classifiers. Similarly, in the $8 \mathrm{TeV}$ analysis the bidimensional distribution of events in the $\left(m_{l l}, m_{T}\right)$ plane is used for different-flavour dilepton channels with zero and one jet; $m_{l l}$ is the invariant mass of the dilepton pair, and $m_{T}$ is the transverse mass reconstructed from the transverse momentum of the dilepton and the missing transverse momentum. Using the bidimensional distributions allowed to improve significantly the sensitivity of the analysis as compared to the previous one made with $5 \mathrm{fb}^{-1}$ of $8 \mathrm{TeV}$ data. All background rates, except for very small contributions from $\mathrm{WZ}, \mathrm{ZZ}$, and $\mathrm{W} \gamma$, are evaluated from data.

In the $H \rightarrow Z Z \rightarrow 2 \ell 2 \tau$ search [11], one $Z$ boson is required to be on-shell and to decay to a lepton pair (ee or $\mu \mu)$. The other $Z$ boson is required to decay through a $\tau \tau$ pair to one of the four final-state signatures $e \mu, e \tau_{\mathrm{h}}, \mu \tau_{\mathrm{h}}, \tau_{\mathrm{h}} \tau_{\mathrm{h}}$. Thus, eight exclusive sub-channels are defined. We search for a broad excess in the distribution of the dilepton-ditau mass, constructed from the visible products of the tau decays, neglecting the effect of the accompanying neutrinos. The dominant background is non-resonant $Z Z$ production whose rate is estimated from simulation. The main sub-leading backgrounds with jets misidentified as $\tau$ leptons stem from $Z+$ jets (including $Z W$ ) and top-quark events. These backgrounds are estimated from data.

In the $H \rightarrow Z Z^{(*)} \rightarrow 4 \ell$ channel [11], we search for a four-lepton mass peak over a small continuum background. This is extremely clean, high resolution channel, often called "Golden", but with a very low rate. For this reason high trigger and selection efficiencies are very important. Collision events are selected by the trigger system that requires the presence of a pair of electrons or a pair of muons, or a triplet of electrons. A trigger requiring an electron and a muon is also used for the 2012 data. The trigger efficiency is higher than $98 \%$. To further separate signal and background, a matrix
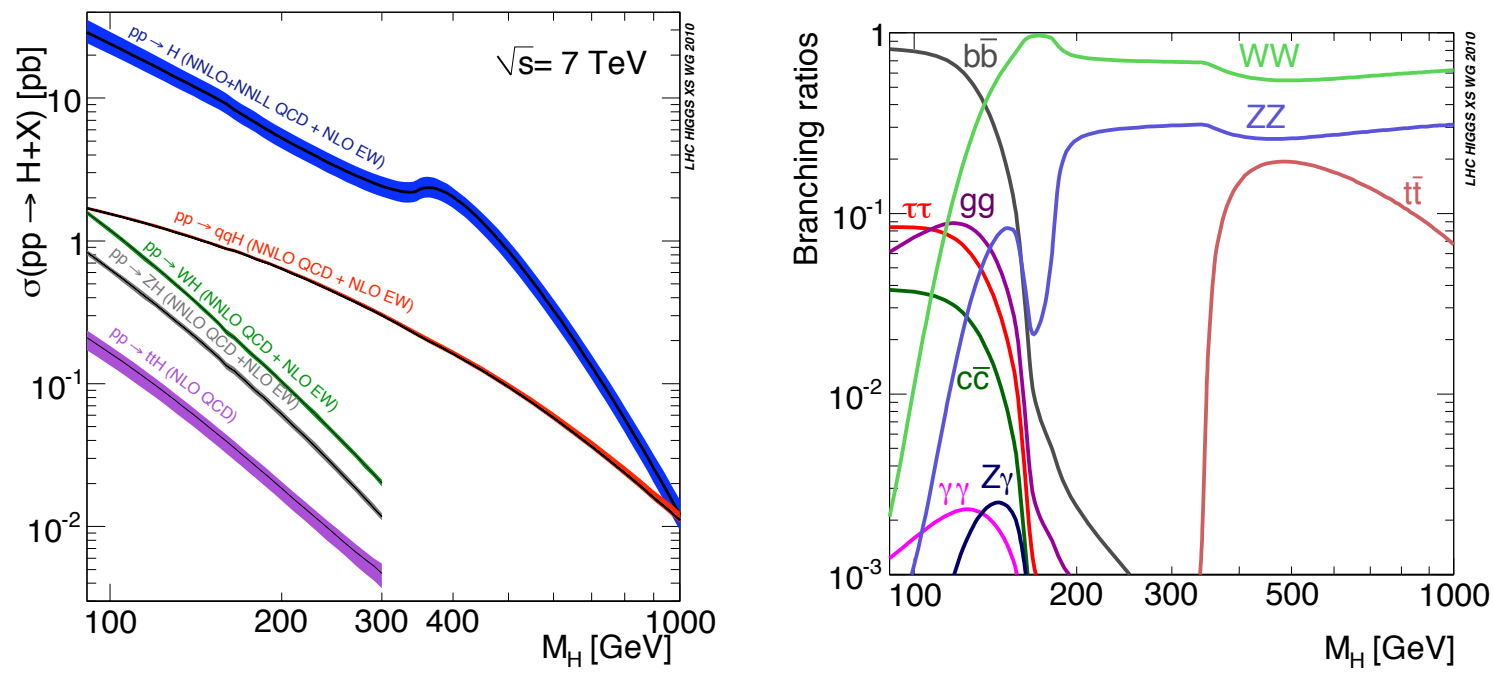

Figure 1: The standard model Higgs boson production cross sections (left) and decay branching fractions (right). The plots are taken from Ref. [9]. 
Table 1: Summary of the SM Higgs boson decay channels and production modes relevant for the searches and studies at the LHC

\begin{tabular}{|c|c|c|c|c|c|c|c|}
\hline Channel & $\begin{array}{l}\text { Masses } \\
{[\mathrm{GeV}]}\end{array}$ & $\begin{array}{l}\text { Branch. } \\
\text { ratio }\end{array}$ & $\begin{array}{c}N_{e v} \\
10 \mathrm{fb}^{-1}\end{array}$ & $\begin{array}{l}\text { Prod. } \\
\text { modes }\end{array}$ & $\mathrm{S} / \mathrm{B}$ & $\begin{array}{l}\text { Mass } \\
\text { res. }\end{array}$ & Remark \\
\hline$H \rightarrow \gamma \gamma$ & $110-150$ & $\begin{array}{c}2 \\
\times 10^{-3}\end{array}$ & $200 / 400$ & $\begin{array}{l}\text { inclusive, } \\
\text { VBF }\end{array}$ & $\begin{array}{c}\text { low } \\
\mathrm{O}(0.1)\end{array}$ & $2 \%$ & $\begin{array}{l}\text { max. sens. } \\
\text { at low mass }\end{array}$ \\
\hline$H \rightarrow b b$ & $110-135$ & 0.7 & $30 / 10^{5}$ & $\mathrm{VH}$ & $\begin{array}{c}\text { low } \\
\mathrm{O}(0.1)\end{array}$ & $10 \%$ & \\
\hline$H \rightarrow \tau \tau$ & $110-150$ & 0.08 & $20 / 10^{4}$ & $\begin{array}{l}\text { inclusive, } \\
\text { VBF, VH }\end{array}$ & $\begin{array}{c}\text { low } \\
\mathrm{O}(0.1)\end{array}$ & $15 \%$ & \\
\hline $\begin{array}{l}H \rightarrow W W\left({ }^{*}\right) \rightarrow \\
\rightarrow l v l v\end{array}$ & $110-700$ & 0.03 & $60 / 10^{3}$ & $\begin{array}{l}\text { gg fusion, } \\
\text { VBF, VH }\end{array}$ & $\begin{array}{c}\text { medium } \\
\mathrm{O}(1)\end{array}$ & - & $\begin{array}{l}\text { max. sens. } \\
\text { at interm. }\end{array}$ \\
\hline $\begin{array}{l}H \rightarrow Z Z\left(^{*}\right) \rightarrow \\
\rightarrow 4 l\end{array}$ & $110-700$ & $\begin{array}{l}(3-8) \\
\times 10^{-4}\end{array}$ & $6 / 20$ & inclusive & $\begin{array}{c}\text { high } \\
>1\end{array}$ & $2 \%$ & \\
\hline $\begin{array}{l}H \rightarrow Z Z \rightarrow \\
\rightarrow \text { other }\end{array}$ & $200-700$ & 0.2 & - & inclusive & & $10 \%$ & $\begin{array}{l}\text { max. sens. } \\
\text { at high mass }\end{array}$ \\
\hline $\begin{array}{l}H \rightarrow W W \rightarrow \\
\rightarrow \operatorname{lv} q q\end{array}$ & $170-700$ & 0.15 & - & inclusive & & & \\
\hline
\end{tabular}

Table 2: Summary of Higgs analyses and documentation

\begin{tabular}{|l|c|c|c|c|}
\hline Channel & $\begin{array}{c}\text { Masses } \\
{[\mathrm{GeV}]}\end{array}$ & $\begin{array}{c}\text { Data used, } f b^{-1} \\
(7+8 \mathrm{TeV})\end{array}$ & References & Remarks \\
\hline$H \rightarrow \gamma \gamma$ & $110-150$ & $5.1+5.3$ & HIG-12-15 & Mass measurement \\
\hline$H \rightarrow b b$ & $110-135$ & $5+12.1$ & HIG-12-44 & \\
\hline$H \rightarrow \tau \tau$ & $110-145$ & $4.9+12.1$ & HIG-12-43 & \\
\hline$H \rightarrow W W\left(^{*}\right) \rightarrow l v l \nu$ & $110-700$ & $4.9+12.1$ & HIG-12-42 & \\
\hline$H \rightarrow Z Z\left(^{*}\right) \rightarrow 4 l$ & $110-700$ & $5.1+12.2$ & HIG-12-41 & Mass and parity measurements \\
\hline \hline Higgs combination & $110-700$ & $4.9-5.1+5.3-12.2$ & HIG-12-45 & \\
\hline$H \rightarrow W W \rightarrow l v q q$ & $170-700$ & $5+12$ & HIG-12-46 & \\
\hline
\end{tabular}

element likelihood analysis (MELA) is used. Five angles $\Theta^{*}, \Phi_{1}, \Theta_{1}, \Theta_{2}, \Phi$ defined in [12], and the invariant masses of the lepton pairs, $m_{Z_{1}}$ and $m_{Z_{2}}$, fully describe the kinematics of the $H \rightarrow Z Z \rightarrow 4 l$ process at a given mass of the four-lepton system in their centre-of-mass frame. These observables provide significant discriminating power between signal and background. We construct a kinematic discriminant $\left(K_{D}\right)$ based on the probability ratio of the signal and background hypotheses, MELA $K_{D}=P_{s i g} /\left(P_{s i g}+P_{b k g}\right)$, as described in [12], [13]. The likelihood ratio is defined for each value of $m_{4 l}$. The $4 e, 4 \mu, 2 e 2 \mu$ sub-channels are analyzed separately since there are differences in the four-lepton mass resolutions and the background rates arising from jets misidentified as leptons. The dominant irreducible background in this channel is from non-resonant $Z Z$ production (with both $Z$ bosons decaying to either $2 e$, or $2 \mu$, or $2 \tau$ with the taus decaying leptonically) and is estimated from simulation. The smaller reducible backgrounds with jets misidentified as leptons, e.g. 
$Z+$ jets, are estimated from data. This analysis (including $2 \ell 2 \tau$ ) excludes the SM Higgs boson at $95 \% \mathrm{CL}$. in the ranges $113<m_{H}<116 \mathrm{GeV}, 129<m_{H}<720 \mathrm{GeV}$. The local significance of the excess at 125.5 is $4.4 \sigma$ (expected for the SM Higgs $5 \sigma$ ). The best fit signal strength at $125 \mathrm{GeV}$ is $0.74 \pm 0.25$. The mass fit gives $126.2 \pm 0.6$ (stat) \pm 0.2 (syst) GeV.

The statistics used in the analysis $H \rightarrow Z Z^{(*)} \rightarrow 4 \ell$, significantly increased w.r.t. the previous result [6], allowed to perform for the first time the parity measurement of the discovered boson. Here we follow the MELA methodology, where instead of signal-to-background probability ratio we construct probability ratio for two signal hypotheses. The data disfavors the pseudoscalar hypothesis $0^{-}$with a $C L_{s}$ value of $2.4 \%$.

The $H \rightarrow \gamma \gamma$ analysis [6], [14] is focused on a search for a narrow peak in the diphoton mass distribution. This is the most important channel in the low mass range. The event sample is split into two mutually exclusive classes: (i) diphoton events with one forward and one backward jet, consistent with the VBF topology, and (ii) all remaining events. This division is motivated by the consideration that there is a better signal-to-background ratio in the first class than in the second. For the $8 \mathrm{TeV}$ data, the dijet class is split into two sub-classes: events with low and high dijet mass $m_{j j}$. The non-dijet class, containing over $99 \%$ of the data, is subdivided into four sub-classes based on the output of a multivariate discriminant that assigns a high score to signal-like events, based on (i) an event-by-event estimate of the diphoton mass resolution, (ii) a photon identification score for each photon, and (iii) kinematic information about the photons and the diphoton system. The photon identification score is obtained from a multivariate analysis (MVA) discriminant that uses shower shape information and isolation variables to separate prompt photons from those arising from jets. The background in the signal region is estimated from a fit to the observed diphoton mass distribution in data. This analysis alone observes the excess of events above the expected standard model background for a Higgs boson mass hypothesis of $125 \mathrm{GeV}$ with a local significance of 4.1 $\sigma$ (expected from the SM Higgs boson $2.6 \sigma$ ). The best fit signal strength at $125 \mathrm{GeV}$ is $1.56 \pm$ 0.43 .

The $H \rightarrow \tau \tau$ search [15] is performed using the final-state signatures $e \mu, \mu \mu, e \tau_{\mathrm{h}}$, and $\mu \tau_{\mathrm{h}}$, where electrons and muons arise from leptonic $\tau$-decays $\tau \rightarrow \ell v_{\ell} v_{\tau}$ and $\tau_{\mathrm{h}}$ denotes hadronic $\tau$ decays $\tau \rightarrow$ hadrons $+v_{\tau}$. Each of these categories is further divided into three exclusive subcategories based on the number and the type of the jets in the event: (i) events with one forward and one backward jet, consistent with the VBF topology, (ii) events with at least one high $p_{T}$ hadronic jet but not selected in the previous category, and (iii) events with one jet with a small $p_{T}$. The last two categories are further divided by the transverse momentum of the visible tau decay. In each of these categories, we search for a broad excess in the reconstructed $\tau \tau$ mass distribution. The main irreducible background, $Z \rightarrow \tau \tau$ production, and the largest reducible backgrounds (W+ jets, multijet production, $Z \rightarrow e e$ ) are evaluated from various control samples in data. The treatment of the VBF category has been changed with respect to Ref. [6]. The observed upper limit on the higgs production at $125 \mathrm{GeV}$ is 1.63 of the Standard Model prediction while the expected limit is on the level of the Standard Model prediction.

The search for $H \rightarrow \tau \tau$ decays produced in association with a $\mathrm{W}$ or $\mathrm{Z}$ boson is conducted in events with three or four leptons in the final state [16] The WH analysis selects events which have same charged electrons or muons and a hadronically-decaying tau: $e^{+} e^{+} \tau_{\mathrm{h}}^{-}$and $\mu^{+} \mu^{+} \tau_{\mathrm{h}}^{-}$. The $\mathrm{ZH}$ analysis is performed in events with an identified $Z \rightarrow e e$ or $Z \rightarrow \mu \mu$ decay and a Higgs boson 
candidate with one of the following final states: $e \mu, e \tau_{\mathrm{h}}, \mu \tau_{\mathrm{h}}, \tau_{\mathrm{h}} \tau_{\mathrm{h}}$. The main irreducible backgrounds to the $\mathrm{WH}$ and $\mathrm{ZH}$ searches are $\mathrm{WZ}$ and $\mathrm{ZZ}$ diboson events, respectively. The irreducible backgrounds are estimated using simulation, corrected by control samples in data. The reducible backgrounds in both analyses are $\mathrm{W}, \mathrm{Z}$, and $t \bar{t}$ events with at least one quark or gluon jet misidentified as an isolated $e, \mu$, or $\tau_{\mathrm{h}}$. These backgrounds are estimated solely from data by measuring the probability for jets to be misidentified as isolated leptons in background-enriched control regions, and weighting the selected events which fail the lepton requirements by the misidentification probability.

The $H \rightarrow b b$ search [17] concentrates on Higgs boson production in association with $W$ or $Z$ bosons, in which the focus is on the following decay modes: $W \rightarrow e v / \mu v$ and $Z \rightarrow e e / \mu \mu / v v$. The $Z \rightarrow v v$ decay is identified by requiring a large missing transverse energy $E_{T}^{\text {miss }}$ computed from the vector sum of the transverse momenta of all reconstructed objects in the detector (leptons, photons, and charged/neutral hadrons) which are not found to arise from additional interactions. The Higgs boson candidate is reconstructed by requiring two b-tagged jets [18]. The search is divided into events where the vector bosons have medium or large transverse momentum and recoil away from the candidate Higgs boson. A multivariate regression algorithm to better estimate the b-jet $p_{T}$ is trained on jets in simulated signal events and achieves a final dijet mass resolution of $8-9 \%$ for $m_{H}=125 \mathrm{GeV}$. The performance of the regression algorithm is checked in data using $\mathrm{W}$, $\mathrm{Z}+\mathrm{jets}$ and $t \bar{t}$ events. Events with higher transverse momentum bosons have smaller backgrounds and a better dijet mass resolution. In the $8 \mathrm{TeV}$ analysis, for all final states except $Z \rightarrow l l$, the large transverse momentum events are further divided depending on whether they satisfy tight or loose b-tagging requirements. A multivariate analysis technique, trained on simulated signal and background events for several different values of the Higgs boson mass, is used to separate signal and background events. The rates of the main backgrounds, consisting of $\mathrm{W} / \mathrm{Z}+$ jets and top-quark events, are derived from signal-depleted control samples in data. The WZ and ZZ backgrounds with a $\mathrm{Z}$ boson decaying to a pair of b-quarks, as well as the single-top background, are estimated from simulation. The MVA output distribution is used as the final discriminant in the limit setting. This analysis observes the $2.2 \sigma$ excess of events over the background, consistent with the SM Higgs boson expectation.

\section{Combination methodology}

The combination of the SM Higgs boson searches requires simultaneous analysis of the data from all individual search channels, accounting for all statistical and systematic uncertainties and their correlations. The results presented here are based on a combination of Higgs boson searches in the exclusive sub-channels described in Section 4. Depending on the sub-channel, the input to the combination may be a total number of selected events or an event distribution for the final discriminating variable. Either binned or unbinned distributions are used, depending upon the particular search sub-channel.

The number of sources of systematic uncertainties considered in the combination depends on the Higgs boson mass, exceeding 200 for some masses. A large fraction of these uncertainties are correlated across different channels and between signal and backgrounds within a given channel. Uncertainties considered include: theoretical uncertainties on the expected cross sections and ac- 
ceptances for signal and background processes, experimental uncertainties arising from modelling of the detector response (event reconstruction and selection efficiencies, energy scale and resolution), and statistical uncertainties associated with either ancillary measurements of backgrounds in control regions or selection efficiencies obtained using simulated events. Systematic uncertainties can affect either the shape of distributions, or event yields, or both.

The combination is repeated for more than 200 Higgs boson mass hypotheses in the range $110-1000 \mathrm{GeV}$. The step size in this scan varies across the mass range and is determined by the Higgs boson mass resolution. The minimum step size is $0.5 \mathrm{GeV}$ at lower masses, where it is commensurate with the mass resolution of the $\gamma \gamma$ and $4 l$ channels. The maximum step size is tens of $\mathrm{GeV}$ at large masses, where the intrinsic Higgs boson width is the limiting factor.

The overall statistical methodology used in this combination was developed by the CMS and ATLAS collaborations in the context of the LHC Higgs Combination Group. The detailed description of the methodology can be found in [19], [20]. Results presented in this note are obtained using asymptotic formulae [21], including a few updates recently introduced in the RooStats package [22].

The combination procedure introduces a signal strength modifier $\mu$ that multiplies the expected SM Higgs boson cross section such that $\sigma=\mu \cdot \sigma_{\mathrm{SM}}$. Each independent source of systematic uncertainty is assigned a nuisance parameter $\theta_{i}$. The expected Higgs boson and background yields are functions of these nuisance parameters, and are written as $\mu \cdot s(\theta)$ and $b(\theta)$, respectively. Most nuisance parameters are constrained by other measurements. They are encoded in the probability density functions $p_{i}\left(\tilde{\theta}_{i} \mid \theta_{i}\right)$ describing the probability to measure a value $\tilde{\theta}_{i}$ of the $i$-th nuisance parameter, given its true value $\theta_{i}$.

For the calculation of the exclusion limit, we adopt the modified frequentist construction $C L_{s}$ [23].

$$
C L_{s}=\frac{\mathrm{CL}_{\mathrm{s}+\mathrm{b}}}{\mathrm{CL}_{\mathrm{b}}} .
$$

If $C L_{s} \leq \alpha$ for $\mu=1$, we determine that the SM Higgs boson is excluded at the $1-\alpha$ confidence level. To quote the upper limit on $\mu$ at the $95 \%$ confidence level, we adjust $\mu$ until we reach $C L_{s}=0.05$.

\section{Results}

Combined results of searches for SM Higgs bosons are presented in this section. Unless stated otherwise, the following conventions are used. The observed values are shown by a solid line. A dashed line indicates the median of the expected results for the background-only hypothesis. The green (dark) and yellow (light) bands indicate the ranges in which the measured values are expected to reside in at least $68 \%$ and $95 \%$ of all experiments under the background-only hypothesis. The probabilities for an observation to lie above or below the 68\% (95\%) band are at most $16 \%(2.5 \%)$ each. The observed and median expected values of $C L_{s}$ as well as the $68 \%$ and $95 \%$ bands are obtained by generating ensembles of pseudo-datasets.

The mass regions where the observed $C L_{s}$ values are smaller than 0.05 are excluded at $95 \%$ $\mathrm{CL}$, the regions where they are smaller than 0.01 are excluded at 99\% CL. The median expected exclusion range of $m_{H}$ at $95 \% \mathrm{CL}$ in the absence of a signal is $110-650 \mathrm{GeV}$. For the largest 

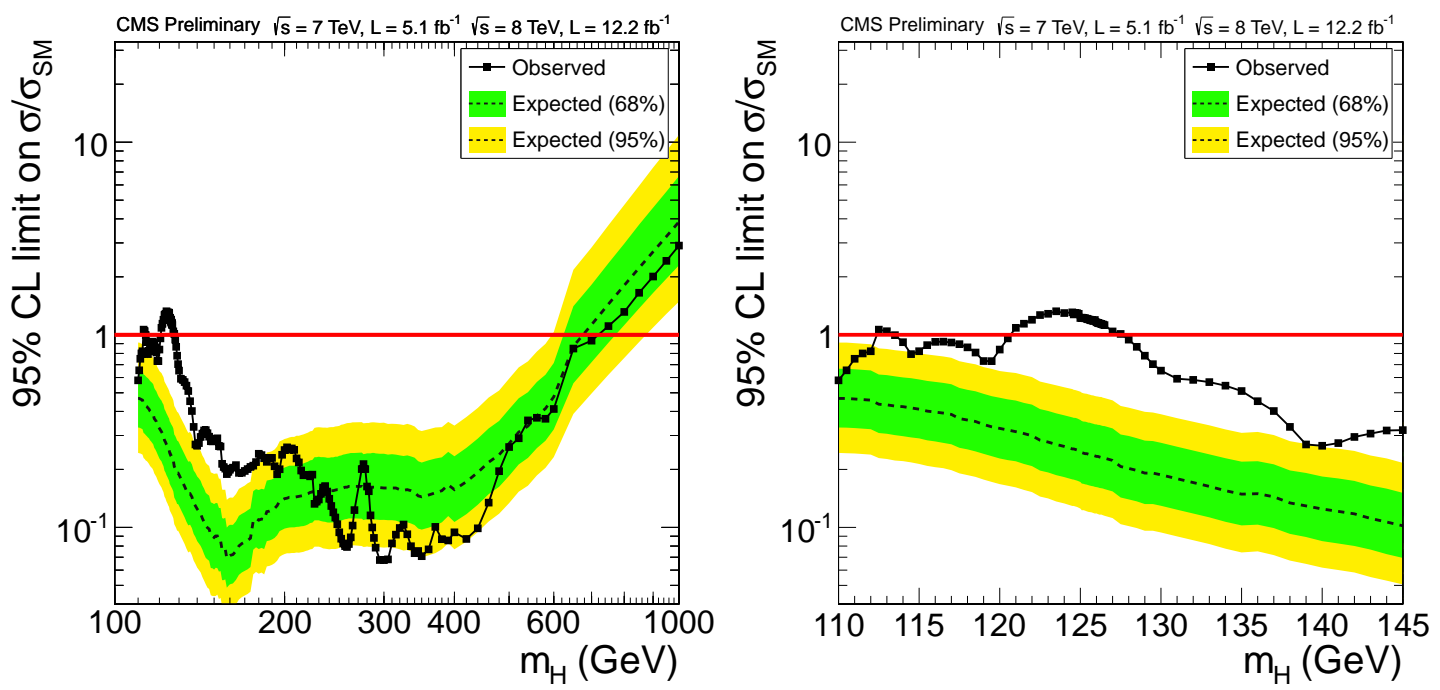

Figure 2: The observed and expected 95\% CL upper limits on the signal strength parameter $\mu=\sigma / \sigma_{\mathrm{SM}}$ for the SM Higgs boson hypothesis as a function of the Higgs boson mass in the range 110-1000 GeV (left) and $110-145 \mathrm{GeV}$ (right).

values of $m_{H}$, we observe fewer events than the median expected number for the background-only hypothesis, which makes the observed limits in that range stronger than expected. At small $m_{H}$ we observe an excess of events due to the recently discovered new boson. This makes the observed limits weaker than expected in the absence of a SM Higgs boson. We exclude a SM Higgs boson at $95 \% \mathrm{CL}$ in the mass range $127.5-700 \mathrm{GeV}$.

Figure 2 shows the $95 \%$ CL upper limits on the signal strength modifier, $\mu=\sigma / \sigma_{\mathrm{SM}}$, obtained by generating ensembles of pseudo-datasets, as a function of $m_{H}$. The ordinate thus shows the Higgs boson cross section that is excluded at $95 \% \mathrm{CL}$, expressed as a multiple of the SM Higgs boson cross section.

To quantify the inconsistency of the observed excesses with the background-only hypothesis, we show in Fig. 3 (left) a scan of the combined local $p$-value $p_{0}$ in the low-mass region. The local $p$-values shown in Fig. 3 are obtained with the asymptotic formula. They reach the values corresponding to $6.9 \sigma$.

The $p$-value characterises the probability of background producing an observed excess of events, but it does not give information about the compatibility of an excess with an expected signal. The latter is provided by the best fit $\hat{\mu}$ value, shown in Fig. 3 (right).

\section{Mass measurements}

To measure the mass of the observed state, we use the $Z Z \rightarrow 4 l$ and $\gamma \gamma$ channels that have excellent mass resolution and for which we observe excesses with significances of $4.4 \sigma$ and 4.0 $\sigma$, respectively. Fig. 4 (left) shows 2D 68\% confidence level regions for the two parameters of interest, the signal strength modifier $\mathrm{m}$ and the mass $\mathrm{mX}$, for these channels. The combined $68 \%$ CL contour shown with a black line in Fig. 4 (left) fixes the relative event yield between the two 

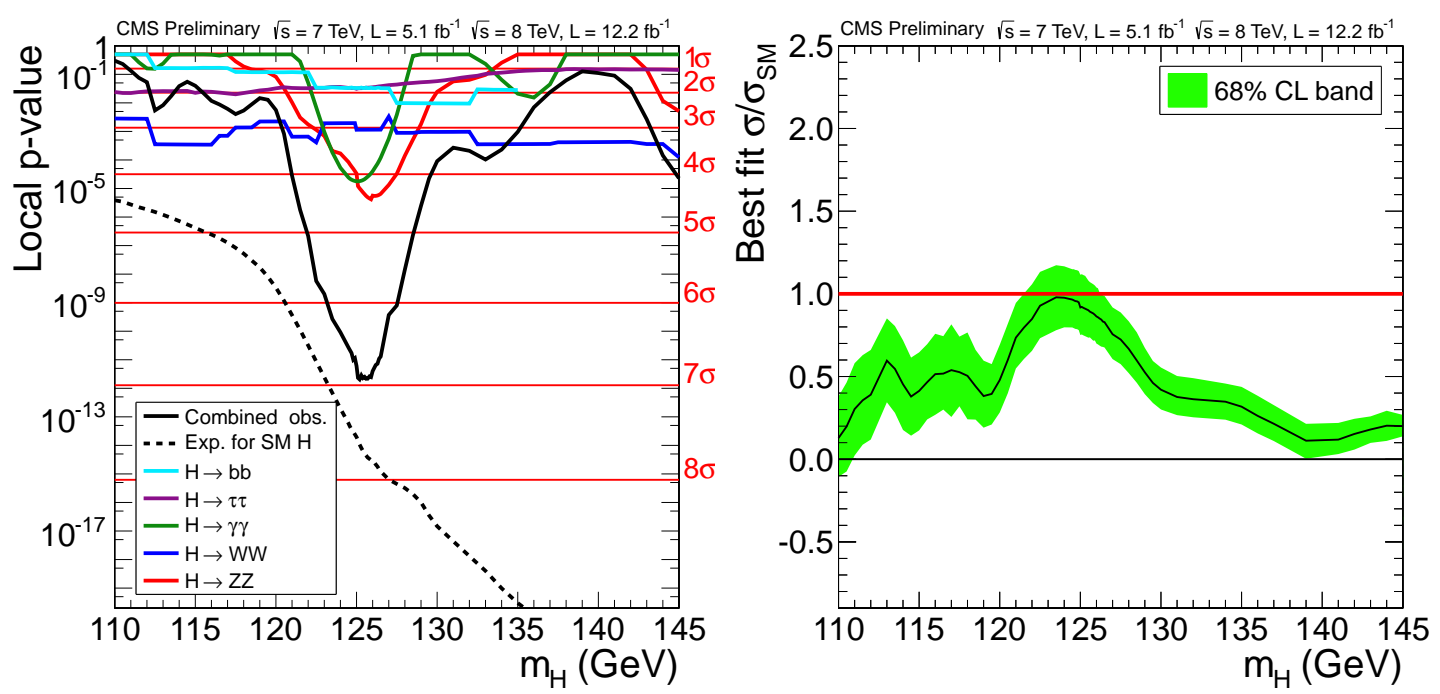

Figure 3: The observed local $p$-value $p_{0}$ (left) and best-fit $\hat{\mu}=\sigma / \sigma_{\mathrm{SM}}$ (right) as a function of the SM Higgs boson mass in the range 110-145 GeV. The local $p$-values for individual channels and their combination are obtained with the asymptotic formula (lines); the combined local $p$-value is validated by generating ensembles of background-only pseudo-datasets (points). The dashed line shows the expected local $p$-values $p_{0}\left(m_{H}\right)$, should a Higgs boson with a mass $m H$ exist. The band in the right plot corresponds to the $\pm 1 \sigma$ uncertainties on the $\hat{\mu}$ values.

channels to the SM Higgs boson expectation, while the overall signal strength is left as a free parameter.

To extract the value of $m_{X}$ in a model-independent way, the signal strength modifiers for the $g g \rightarrow H \rightarrow \gamma \gamma, V B F+V H \rightarrow \gamma \gamma$, and $H \rightarrow Z Z \rightarrow 4 l$ processes are assumed to be independent and, thus, not tied to the SM expectation. The signal in all channels is assumed to be due to a state with a unique mass $m_{X}$. The mass $m_{X}$ and its uncertainty are extracted from a scan of the combined test statistic $q\left(m_{X}\right)$ with the three signal signal strength modifiers profiled in the same way as all other nuisance parameters. Fig. 4 (right) shows the scan of the test statistic as a function of the hypothesised mass $m_{X}$ for the two final states separately and their combination. Crossings of the $q\left(m_{X}\right)$ curves with horizontal thick (thin) lines at 1 (3.8) define the 68\% (95\%) CL intervals for the mass of the observed particle. These intervals include both statistical and systematic uncertainties. The $68 \% \mathrm{CL}$ interval is $m_{X}=125.8 \pm 0.5 \mathrm{GeV}$.

To evaluate the statistical component of the overall error, we also perform a scan of the test statistic $q(m X)$ with all nuisance parameters fixed to their best-fit values. The resulting 68\% CL interval is: $m_{X}=125.8 \pm 0.4$ (stat) GeV. Assuming that the total error is the sum in quadrature of the statistical and systematic components, we extract a systematic error of $0.4 \mathrm{GeV}$. Therefore, the final mass measurement can be written as $m_{X}=125.8 \pm 0.4$ (stat) \pm 0.4 (syst) GeV.

\section{Conclusions}

Results are presented from searches for the standard model (SM) Higgs boson in proton proton collisions at $\sqrt{s}=7$ and $8 \mathrm{TeV}$, using data samples corresponding to integrated luminosities of up 

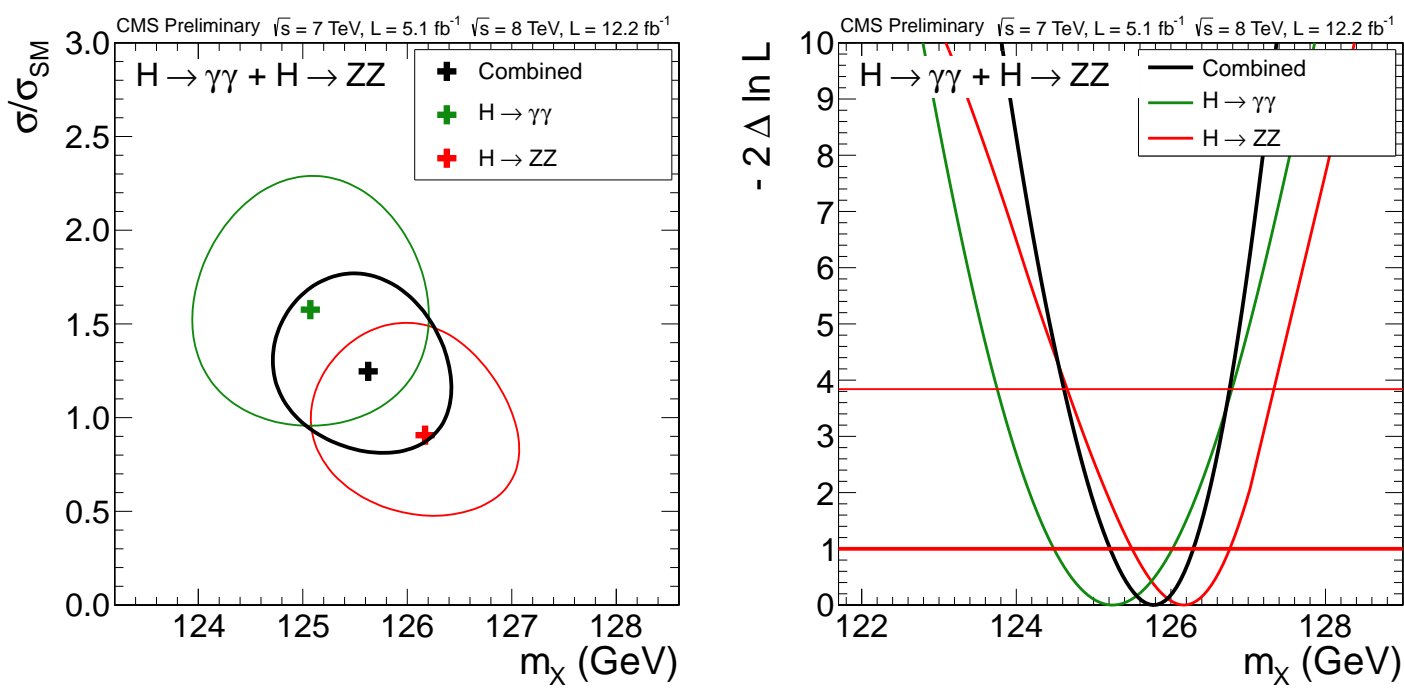

Figure 4: (Left) 2D 68\% CL contours for a hypothesised boson mass $m_{X}$ and signal strength $\mu=\sigma / \sigma_{S M}$ for the $\gamma \gamma$ and $4 l$ final state, and their combination, assuming one common signal strength $\mu$. (Right) 1D-scan of test statistic $q\left(m_{X}\right)$ (or $-2 \Delta \ln L$ ) vs hypothesised boson mass $m_{X}$ for the $\gamma \gamma$ and $4 l$ final states separately and for their combination. In this combination, three independent signal strengths $g g \rightarrow H \rightarrow \gamma \gamma$, $V B F+V H \rightarrow \gamma \gamma$, and $H \rightarrow Z Z \rightarrow 4 l$ are profiled together with all other nuisance parameters.

to $5.1 \mathrm{fb}^{-1}$ at $7 \mathrm{TeV}$ and up to $12.2 \mathrm{fb}^{-1}$ at $8 \mathrm{TeV}$. The search is performed in the mass range 110$1000 \mathrm{GeV}$ in five decay modes: $\gamma \gamma, Z Z, W W, \tau \tau$, and $b b$. No evidence is found for a significant excess of events that could be attributed to an additional particle, beyond the recently observed boson with a mass near $125 \mathrm{GeV}$. The significance of the latter is now $6.9 \sigma$. Its mass is measured to be $125.8 \pm 0.4$ (stat) \pm 0.4 (syst) GeV. The event yields obtained by the different analyses targeting specific decay modes and production mechanisms are consistent with those expected for the SM Higgs boson. The best-fit signal strength for all channels combined, expressed in units of the SM Higgs boson cross section, is $0.88 \pm 0.21$. The consistency of the couplings of the observed boson with those predicted for the SM Higgs boson is tested in various ways, and no significant deviations are found. Under the assumption that the observed boson has spin zero, the data disfavour the psudo-scalar hypothesis $0^{-}$with a $C L_{s}$ value of $2.4 \%$.

\section{References}

[1] S. L. Glashow, "Partial-symmetries of weak interactions" Nucl. Phys. 22 (1961) 579.

[2] S. Weinberg, "A model of leptons" Phys. Rev. Lett. 19 (1967) 1264.

[3] P. W. Higgs, "Broken symmetries, massless particles and gauge fields" Phys. Lett. 12 (1964) 132.

[4] P. W. Higgs, "Broken symmetries and the masses of gauge bosons" Phys. Rev. Lett. 13 (1964) 508.

[5] K. Riesselman, hep-ph/9711456.

[6] CMS Collaboration, "Observation of a new boson at a mass of $125 \mathrm{GeV}$ with the CMS experiment at the LHC", Phys. Lett. B (2012), arXiv:1207.7235. 
[7] ATLAS Collaboration, "Observation of a new particle in the search for the Standard Model Higgs boson with the ATLAS detector at the LHC", Phys. Lett. B (2012), arXiv:1207.7214.

[8] CMS Collaboration, "The CMS experiment at the CERN LHC" JINST 3 (2008) S08004.

[9] LHC Higgs Cross SectionWorking Group Collaboration, "Handbook of LHC Higgs Cross Sections: 1. Inclusive Observables", CERN CERN-2011-002, (2011).

[10] CMS Collaboration, "Evidence for a particle decaying to $\mathrm{W}+\mathrm{W}$ - in the fully leptonic final state in a standard model Higgs boson search in pp collisions at the LHC", CMS Physics Analysis Summary CMS-PAS-HIG-12-042 (2012). Previous published 7 TeV result is in arXiv:1202.1489.

[11] CMS Collaboration, "Updated results on the new boson discovered in the search for the standard model Higgs boson in the $\mathrm{ZZ}$ to 4 leptons channel in pp collisions at $\operatorname{sqrt}(\mathrm{s})=7$ and $8 \mathrm{TeV}$ ", CMS-PAS-HIG-12-041 (2012), CERN preprint: CERN-PH-EP-2012-372

[12] Y. Gao et al., "Spin determination of single-produced resonances at hadron colliders", Phys. Rev. D 81 (2010) 075022, arXiv:1001.3396.

[13] S. Bolognesi, Y. Gao, A. V. Gritsan et al., "On the spin and parity of a single-produced resonance at the LHC", arXiv:1208.4018.

[14] CMS Collaboration, "Search for a standard model Higgs boson decaying into two photons in pp collisions", CMS-PAS-HIG-12-015 (2012)

[15] CMS Collaboration, "Search for standard model Higgs bosons decaying to tau pairs", CMS-PAS-HIG-12-043 (2012).

[16] CMS Collaboration, "Search for a Standard Model Higgs bosons decaying to tau pairs produced in association with a W or a Z boson", CMS-PAS-HIG-12-051 (2012).

[17] CMS Collaboration, "Search for standard model Higgs bosons produced in association with W or Z bosons, and decayng to bottom quarks" CMS-PAS-HIG-12-044 (2012).

[18] CMS Collaboration, "Algorithms for b jet identification in CMS", CMS Physics Analysis Summary CMS-PAS-BTV-09-001 (2009).

[19] ATLAS and CMS Collaborations, LHC Higgs Combination Group, "Procedure for the LHC Higgs boson search combination in Summer 2011", ATL-PHYS-PUB/CMS NOTE 2011-11, 2011/005, (2011).

[20] CMS Collaboration, "Combined results of searches for the standard model Higgs boson in pp collisions at sqrts = 7 TeV", Phys. Lett. B 13 (2012) 26.

[21] G. Cowan et al., "Asymptotic formulae for likelihood-based tests of new physics", Eur. Phys. J. C 71 (2011) 1-19, arXiv:1007.1727.

[22] L. Moneta, K. Belasco, K. S. Cranmer et al., "The RooStats Project", arXiv:1009.1003.

[23] A. L. Read, "Presentation of search results: the CLs technique", J. Phys. G: Nucl. Part. Phys. 28 (2002) 2693. 